\title{
Depth Dose Characteristics of Proton Beams within Therapeutic Energy Range Using the Particle Therapy Simulation Framework (PTSim) Monte Carlo Technique
}

\author{
Siou-Yin Cai ${ }^{1}$, Tsi-Chain Chao ${ }^{1,2}$, Mei-Jyun Lin ${ }^{1,3}$, Chuan-Jung Tung ${ }^{1,2}$, Chung-Chi Lee ${ }^{1,2,3}$
}

Background: The stopping power and range tables published by the National Institute of Standards and Technology (NIST) were obtained by assuming continuous slowing down approximation (CSDA). This study examined more detail depth dose characteristics of ideal proton beams using the particle therapy simulation framework (PTSim) Monte Carlo technique.

Methods: $\quad$ Simulation for parallel broad field geometry (PBFG) was replaced by the pencil beam geometry (PBG) for improved simulation efficiency. Depth dose distributions (Bragg peak, BP) for beam energies from 69.44 to $230.71 \mathrm{MeV}$ at $5 \mathrm{~mm}$ range interval were obtained. This study used seven parameters, $\mathrm{R}_{\text {peak }}, \mathrm{R}_{90}, \mathrm{R}_{80}, \mathrm{R}_{50}$, full width at half maximum (FWHM), $\mathrm{W}_{80-20}$, and peak-to-entrance ratio to represent BP characteristics. The resulting energy-range relationships were fitted into third order polynomial formulae. In addition, initial beam energy spreads at $0-1 \%(1 \sigma)$ of the mean incident energies at 70,110,150,190, and $230 \mathrm{MeV}$ were added into the simulation to uncover their impact on BP shapes.

Results: The study results reveal deeper penetration, broader FWHM and decreased peak-to-entrance dose ratio at increasing beam energy. Study results for beams with initial energy spreads show that $\mathrm{R}_{80}$ can be a good indicator to characterize initial mean energy. They also suggest FWHM is more sensitive than the width of 80-to-20\% distal fall-off in finding initial energy spread.

Conclusion: Detail depth dose characteristics for monoenergetic proton beams and beams with initial energy spreads within therapeutic energy ranges were reported. These data can serve as a good reference for a clinical practitioner in their daily practice.

(Biomed J 2015;38:408-413)

\section{At a Glance Commentary \\ Scientific background of the subject}

Proton therapy is one of the most advanced and fast growing treatment modality in radiation therapy. The main advantage of using proton beams in cancer therapy lies in their depth dose characteristics (Bragg peak, BP) which deliver compatible dose as conventional photon therapy and almost no dose beyond their treatment ranges. Detailed knowledge of the BP characteristics can provide important insight to their clinical use.

\section{What this study adds to the field}

The National Institute of Standards and Technology (NIST) published stopping power and range tables for commonly used materials. The range tables were obtained assuming continuous slowing down approximation (CSDA) without considering multiple Coulomb scattering and nuclear interaction and, therefore can only provide fixed range data. This study examined more detail depth dose characteristics of ideal proton beams using the PTSim Monte Carlo technique. These data can serve as good reference for a clinical practitioner in their daily practice.

\section{Key words: depth dose, Monte Carlo, particle therapy simulation framework, proton therapy}

$\mathrm{T}^{\mathrm{T}}$ raditionally, radiation therapy treats cancers primarily using photons and electrons generated from a linear accelerator. Since these sparsely ionizing radiations deliver compatible dose to a target and surrounding normal tissues, they require sophisticated beam delivery techniques such as intensity modulated radiotherapy or intensity modulated arc

From the ${ }^{1}$ Department of Medical Imaging and Radiological Sciences, College of Medicine, Chang Gung University, Taoyuan, Taiwan; ${ }^{2}$ Dose Assessment Core Laboratory of Institute for Radiological Research, Chang Gung Memorial Hospital at Linkou, Taoyuan, Taiwan; ${ }^{3}$ Department of Radiation Oncology, Chang Gung Memorial Hospital at Linkou, Chang Gung University College of Medicine, Taoyuan, Taiwan

Received: Sep. 23, 2014; Accepted: Sep. 10, 2015

Correspondence to: Dr. Chung-Chi Lee, Department of Medical Imaging and Radiological Sciences, College of Medicine, Chang Gung University, Taoyuan, Taiwan. 259 Wenhua $1^{\text {st }}$ Rd., Gueishan, Taoyuan 333, Taiwan (ROC). Tel.: 886-3-2118800 ext. 5390; Fax: 886-3-3280797; E-mail: cclee@mail.cgu.edu.tw

DOI: $10.4103 / 2319-4170.167076$ 
therapy to concentrate dose deposition to the target volume and spare nearby healthy structures.

Heavy particles including protons and heavy ions are considered having great potential to further conform high dose to the target volume with their superior depth dose characteristics. The advantage of a therapeutic proton beam lies in its fixed penetration depth (the so-called Bragg peak, BP) inside a human body with minimum excess dose given beyond its penetration depth. Knowledge of depth dose characteristics for therapeutic proton beams is, therefore, essential for successful management of these beams in cancer treatment.

Depth dose characteristic of clinical proton beams depends greatly on beam line design and can vary from facility to facility. The National Institute of Standards and Technology (NIST) published stopping power and range tables for proton energies from $1 \mathrm{keV}$ to $10,000 \mathrm{MeV}$, ${ }^{[1]}$ providing excellent reference in predicting ionization energy loss per unit path length and penetrating power (range) of protons in commonly used materials. The NIST continuous slowing down approximation (CSDA) range tables were generated for monoenergenetic protons under the assumption of CSDA and, therefore, predicted fixed beam ranges. However, physics involved in generating therapeutic proton beams and their consequent beam characteristics of penetrating power inside the human body are far more complex than this simple setting. Three major interaction types namely ionization energy loss, multiple Coulomb scattering, and nonelastic nuclear interaction were listed as the most important interaction mechanisms for protons within therapeutic energy ranges. ${ }^{[2]}$ The NIST range data ignored the latter $\mathrm{two}^{[1]}$ and were insufficient to provide detail depth dose (BP) characteristics. Additional data on detail BP characteristics will provide handy references to the clinical practitioner in their daily practice.

This study tries to establish additional reference data of depth dose characteristics in water using the Monte Carlo (MC) technique for both ideal proton beams and beams with additional initial energy spreads. BPs characterized by parameters including percent dose ranges $(50 \%, 80 \%$, and $90 \%$ ), width of $80-20 \%$ distal fall-off, full width at half maximum (FWHM) of the BP, and peak-to-entrance dose ratio were reported for beam energies from 70 to $230 \mathrm{MeV}$. Effects of initial energy spreads on depth dose characteristics were also reported.

\section{METHODS}

\section{The particle therapy simulation framework Monte Carlo technique}

The particle therapy simulation framework (PTSim $)^{[3]}$ has been developed by the fund from the Core Research for Evolutional Science and Technology of Japan Science and Technology Agency, JST/CREST. It is a specialized MC package developed for beam transport simulation through therapeutic proton beam nozzles and phantoms/ patients. It was developed based on the Geant 4 simulation toolkit ${ }^{[4]}$ and its application to clinical proton beams has been validated by Aso et al. ${ }^{[5,6]}$ In this work, all the PTSim simulations were performed using the 2014 March release that was based on the Geant4 ver. 9.6.p02. The default physics list was chosen so that the standard electromagnetic (EM) process, hadron elastic process, and hadron inelastic process were used. ${ }^{[7]}$ In such a physics list, G4EmStandardPhysics_option3 was implemented for modeling EM process, G4HadronElasticPhysics for elastic process of hadrons, G4HadronPhysicsFTF_BIC for inelastic process of hadrons, G4StoppingPhysics for stopping physics, G4RadioactiveDecayPhysics for radioactive decay, and G4IonBinaryCascadePhysics for inelastic process. The quark-gluon string precompound (QGSP) model was implemented to handle collision of high energy hardrons, the laboratory for high energy physics (LHEP) model was used for sampling elastic scattering between particles except pions, and the binary cascade model was used for inelastic process of hadrons. For study purposes, adoption of the PTSim is not necessary. It is chosen in place of the original Geant 4 toolkit due to the easier user interface for source term definition and dose scoring.

\section{Simulation geometry}

Depth dose distributions of infinitely large parallel proton fields incident perpendicularly to the surface of a water phantom were proposed. To simplify the simulation and reduce the required CPU time, this study replaced direct depth dose scoring for an infinitely large parallel field (parallel broad field geometry [PBFG]) using small scoring volumes along the beam axis by dose scoring for a pencil beam using infinitely large slab detectors (pencil beam geometry [PBG]). This integral depth dose as described by Pedroni et al. ${ }^{[8]}$ represented the total dose integrated over the whole plane perpendicular to the beam at depths. This technique was proven effective in one of our previous publications ${ }^{[9]}$ Physics basis for the success of this technique is simple. Under charged particle equilibrium, energy deposition loss due to particles scattered out of the scoring volumes in PBFG will be compensated by those particles scattered into these volumes, making the overall dose deposition equivalent to dose deposition by a pencil beam into an infinitely large detector. To ensure feasibility of this technique in this study, comparison between (1) Depth doses along the central axis from a point pencil beam at a large scoring volume of $30 \times 30 \times 0.2 \mathrm{~cm}^{3}$ and (2) from a 
large $10 \times 10 \mathrm{~cm}^{2}$ parallel field at a small $1 \times 1 \times 0.2 \mathrm{~cm}^{3}$ scoring volume was performed. To eliminate dependence of the simulated absolute dose on incident proton fluence, this work adopted the units of $\mathrm{MeV} \cdot \mathrm{cm}^{2} / \mathrm{g}$ or $\mathrm{Gy} \bullet \mathrm{cm}^{2} / \mathrm{Gp}$ to represent dose deposition per unit fluence (histories $/ \mathrm{cm}^{2}$ or Giga protons $/ \mathrm{cm}^{2}$ ).

With the validation of our PGB simulation, depth dose simulations with no initial energy spread for monoenergetic beam energies from 69.44 to $230.71 \mathrm{MeV}$ at a $0.5 \mathrm{~cm}$ range interval were conducted. The simulation energies correspond to NIST CSDA ranges from 4 to $33.5 \mathrm{~cm}$. All scoring volumes were set to $30 \times 30 \times 0.2 \mathrm{~cm}^{3}$ for the evaluation. To evaluate the effect of initial energy spread on depth dose distribution, initial energy spread at $0-1 \%(1 \sigma)$ of the mean incident energy were performed for beam energies from 70 to $230 \mathrm{MeV}$ at $40 \mathrm{MeV}$ intervals. For all the simulations, no initial angular and lateral spreads were used.

\section{RESULTS AND DISCUSSION}

Figure 1 shows the depth dose (BP) comparison between the PBFG and the PBG simulations. The simulated pristine BPs are identical to each other when taking simulation uncertainty into consideration. This result validates our assumption of simplified PBG simulation.

\section{Depth dose distributions without initial energy spread}

Figure 2 shows the pristine BPs without any initial beam energy spread for the simulated energies. The absolute doses on the vertical axis are represented by dose deposition per Giga incident protons per unit

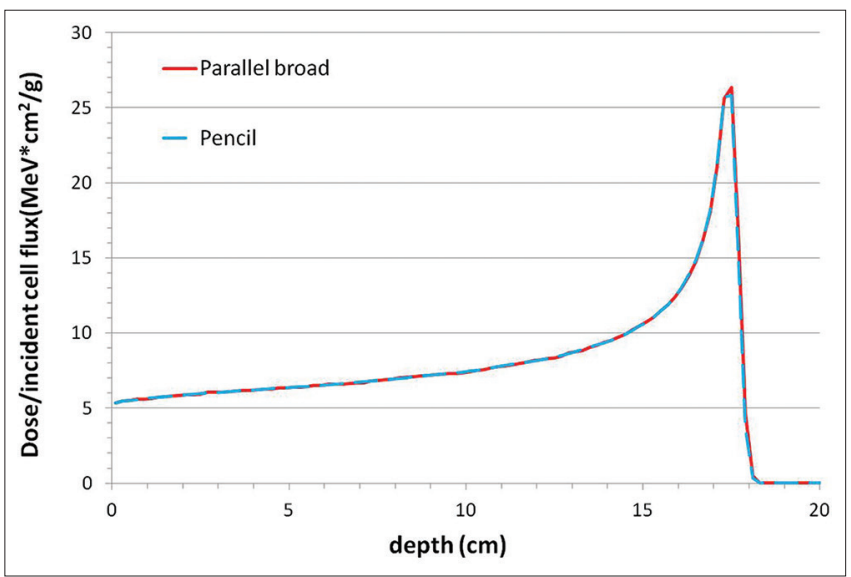

Figure 1: Depth dose comparison between the parallel broad field geometry and the pencil beam geometry simulations for $160 \mathrm{MeV}$ proton beams. For the parallel broad field geometry (Solid red line), field size and scoring volume are $10 \times 10 \mathrm{~cm}^{2}$ and $1 \times 1 \times 0.2 \mathrm{~cm}^{3}$, respectively. For the pencil beam geometry (dashed blue line), field size and scoring volume are $0 \mathrm{~cm}^{2}$ and $30 \times 30 \times 0.2 \mathrm{~cm}^{3}$, respectively. area $\left(\mathrm{Gy} \bullet \mathrm{cm}^{2} / \mathrm{Gp}\right)$. Accumulated effects of the increasing interactions between the incoming protons and the phantom material as the beams penetrate deeper at increasing initial energies can be clearly seen. These effects include decreased surface dose, broadening of the peak FWHM, widening of the distal fall-off (defined as the width between depths of the $80 \%$ and $20 \%$ doses, $\mathrm{W}_{80-20}$ ) and reduction of the peak height. Decrease of the surface dose is primarily due to the decreased stopping power at higher incident beam energy, while broadening of the BPs can be attributed to the combined effect of energy, path length, and angular straggling.

This study uses seven parameters to characterize the shape of a BP: $\mathrm{R}_{\text {peak }}, \mathrm{R}_{90}, \mathrm{R}_{80}, \mathrm{R}_{50}, \mathrm{FWHM}, \mathrm{W}_{80-20}$ and peak-to-entrance dose ratio. $\mathrm{R}_{\mathrm{x}}$ represents depth of the distal $\mathrm{x} \%$ dose point when normalized at its peak value and $R_{\text {peak }}$ is the depth of the peak. Figure $3 A$ shows the plots of the range-energy relationships for $\mathrm{R}_{\mathrm{CSDA}}, \mathrm{R}_{\text {peak }}$, $\mathrm{R}_{90}, \mathrm{R}_{80}$, and $\mathrm{R}_{50}$. Fitting of the data to third-order polynomial formulae is listed in Table 1. Figure 3B summarizes discrepancies $\left(\mathrm{R}_{\mathrm{CSDA}}-\mathrm{R}_{\mathrm{x}}\right)$ between the $\mathrm{MC}$ calculation and the CSDA data for these parameters. The discrepancies should be interpreted with caution because they are not solely due to different physics effects involved in the calculations (e.g. The CSDA approximation ignores the multiple Coulomb scattering process.), but also from uncertainties in stopping power calculation formulae used in the Geant 4 and the NIST calculations. Figure 4 shows the decreasing peak-to-entrance dose ratios as the incident beam energy increases. The decreasing trend is the combined effects of decreasing stopping powers at the phantom surface and reduced peak heights due to BP broadening at higher beam energies. FWHM and $\mathrm{W}_{80-20}$ in Figure 5 demonstrate degradation of the BPs due to increasing

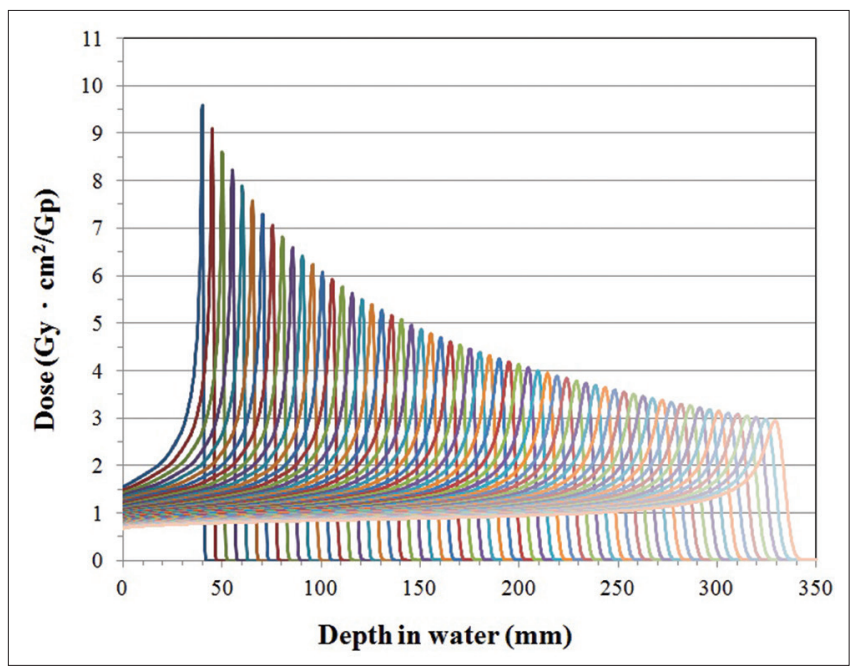

Figure 2: Depth dose curves in water for monoenergetic beam energies from 69.44 to $230.71 \mathrm{MeV}$ at $5 \mathrm{~mm}$ range interval. The dose was represented in absolute unit and Gp stands for $10^{9}$ protons. 

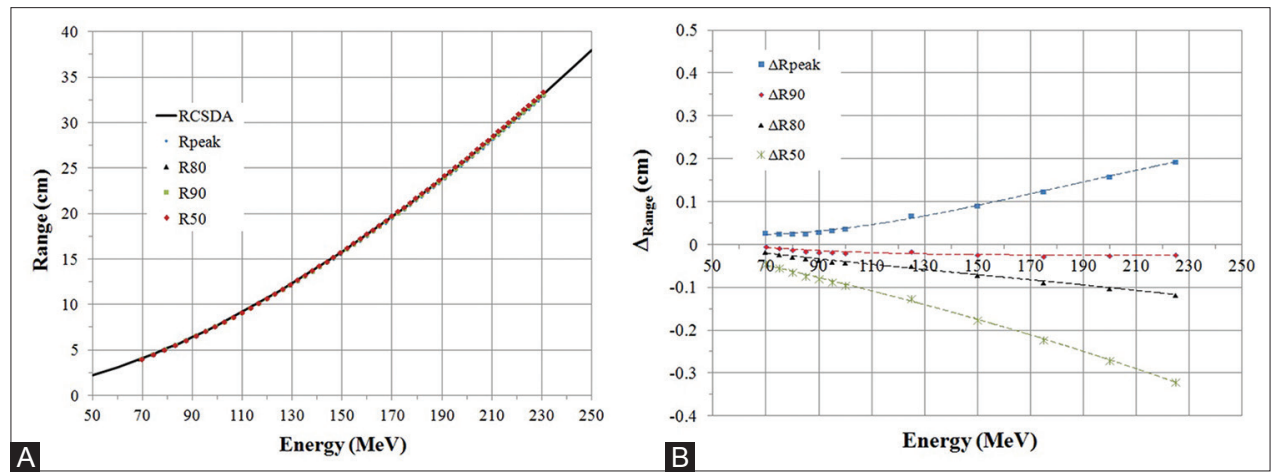

Figure 3: (A) Plots of characteristic beam range parameters $\mathrm{R}_{50}, \mathrm{R}_{80}, \mathrm{R}_{90}$, and $\mathrm{R}_{\text {peak }}$ by particle therapy simulation framework simulation and $\mathrm{R}_{C S D A}$ from National Institute of Standards and Technology PSTAR tables for the depth dose curves in Figure 2. (B) Deviations of the characteristic beam range parameters by particle therapy simulation framework from the $\mathrm{R}_{\mathrm{CSDA}}$ by National Institute of Standards and Technology in Figure $3 \mathrm{~A}$.

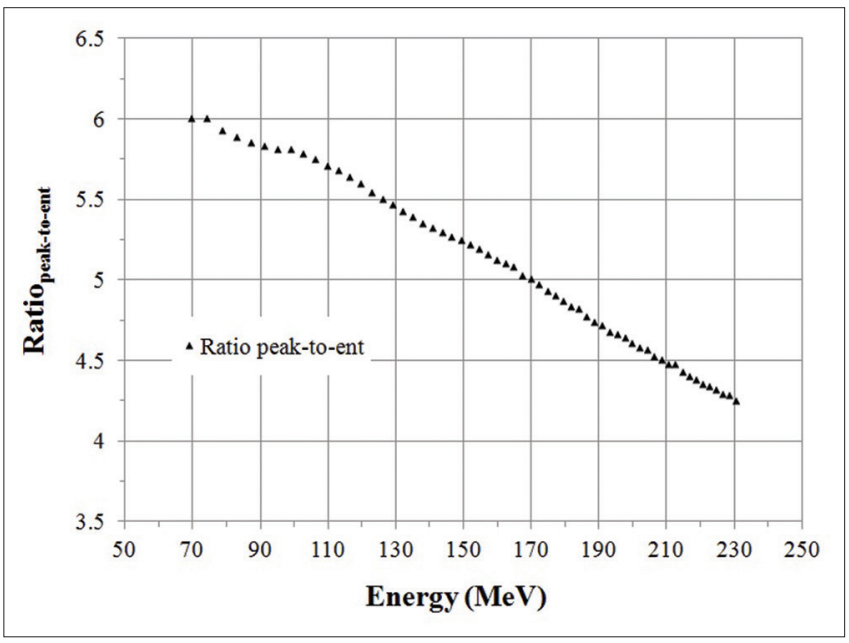

Figure 4: Plots of peak-to-entrance dose ratio for the depth dose curves in Figure 2.

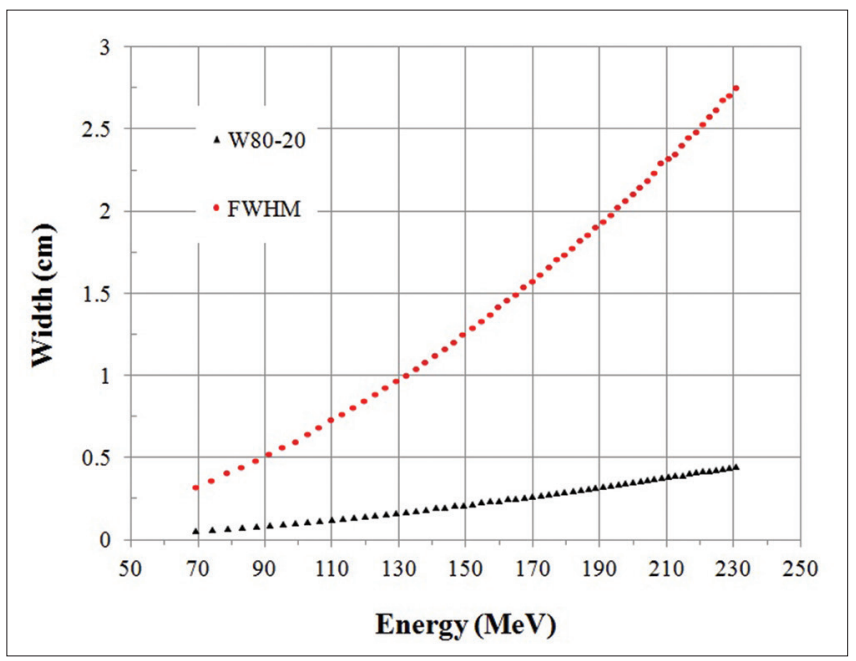

Figure 5: Plots of the full width at half maximum and width of distal $80 \%$ to $20 \%$ dose fall-off for the depth dose curves in Figure 2.

straggling of energy, angle and range as the incident beam energies increase. According to the simulation result, distal
Table 1: Third-order formulae for fitting PTSim simulated data of the characteristics range parameters to beam energy

\begin{tabular}{llc}
\hline Curve type & Fitting formula & $R^{2}$ \\
\hline $\mathrm{R}_{\text {peak }}$ & $\mathrm{y}=-4.813 \mathrm{E}-07 \mathrm{x}^{3}+6.416 \mathrm{E}-04 \mathrm{x}^{2}+2.241 \mathrm{E}-02 \mathrm{x}-4.940 \mathrm{E}-01$ & 1.000 \\
$\mathrm{R}_{90}$ & $\mathrm{y}=-5.033 \mathrm{E}-07 \mathrm{x}^{3}+6.534 \mathrm{E}-04 \mathrm{x}^{2}+2.170 \mathrm{E}-02 \mathrm{x}-4.639 \mathrm{E}-01$ & 1.000 \\
$\mathrm{R}_{80}$ & $\mathrm{y}=-5.059 \mathrm{E}-07 \mathrm{x}^{3}+6.557 \mathrm{E}-04 \mathrm{x}^{2}+2.172 \mathrm{E}-02 \mathrm{x}-4.631 \mathrm{E}-01$ & 1.000 \\
$\mathrm{R}_{50}$ & $\mathrm{y}=-5.101 \mathrm{E}-07 \mathrm{x}^{3}+6.601 \mathrm{E}-04 \mathrm{x}^{2}+2.186 \mathrm{E}-02 \mathrm{x}-4.647 \mathrm{E}-01$ & 1.000 \\
\hline
\end{tabular}

Abbreviations: PTSim: Particle therapy simulation framework; y: Width in $\mathrm{cm}$; $\mathrm{x}$ : Energy in $\mathrm{MeV}$

fall-offs $\left(\mathrm{W}_{80-20}\right.$, in $\left.\mathrm{mm}\right)$ are among the values of $1-2 \%$ of the incident beam energies in $\mathrm{MeV}$. Fitting of the data to third-order polynomial formulae is listed in Table 2 . Closer observation also reveals that FWHM variation on incident beam energy (represented by slope of the curve in Figure 5) is more sensitive than variation of the distal fall-off, indicating FWHM may be a better index in optimizing magnitude of the initial beam energy spread when performing MC simulation for beam transport through a therapeutic beam nozzle.

\section{Depth dose distributions with initial energy spread}

Figure 6 shows the effect of added initial energy spreads to the pristine BPs for five incident beam energies (70-230 at $40 \mathrm{MeV}$ intervals). As expected, they dramatically broaden the peaks and the degree of broadening depends on the magnitudes of the added initial energy spreads. For typical clinical proton beams, this value varies largely due to different accelerator type, energy selection system, and beam line design. Paganetti et al. reported the initial energy spread at the treatment head entrance of a typical cyclotron-based facility is roughly $1 \%$ of its mean energy ${ }^{[10]}$

Figure 7 shows the BPs for $230 \mathrm{MeV}$ beams with initial energy spreads from $0-1 \%$ (at $0.2 \%$ interval). The same BP broadening as in Figure 6 can be observed. When taking the 


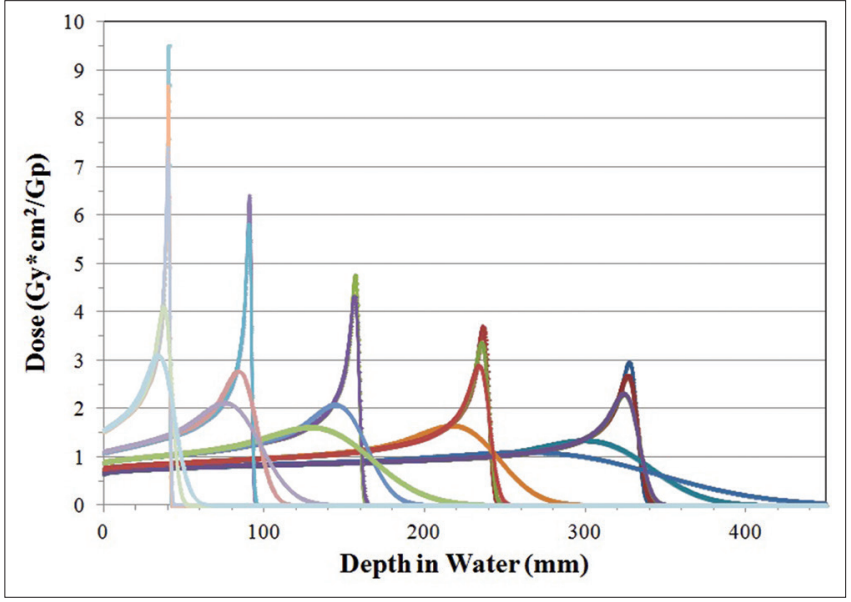

Figure 6: Depth dose curves for proton beams with initial energy spreads. Mean incident energies are 70, 110, 150, 190, and $230 \mathrm{MeV}$. Initial energy spreads $(1 \sigma)$ were set to $0 \%, 0.5 \%, 1 \%, 5 \%$, and $10 \%$ of the mean energies. Broadening of the peaks with increasing initial energy spreads can be clearly seen.

Table 2: Third-order formulae for fitting PTSim simulated data of the $\mathrm{W}_{20-80}$ and FWHM to beam energy

Curve type Fitting formula $R^{2}$

W20-80 $y=-1.007 \mathrm{E}-08 \mathrm{x}^{3}+9.891 \mathrm{E}-06 \mathrm{x}^{2}+2.131 \mathrm{E}-04 \mathrm{x}+4.757 \mathrm{E}-041.000$ FWHM $\quad \mathrm{y}=2.785 \mathrm{E}-08 \mathrm{x}^{3}+2.944 \mathrm{E}-05 \mathrm{x}^{2}+4.186 \mathrm{E}-03 \mathrm{x} 1.210 \mathrm{E}-011.000$

Abbreviations: PTSim: Particle therapy simulation framework; $y$ : Width in $\mathrm{cm}$; $\mathrm{x}$ : Energy in MeV; FWHM: Full width at half maximum

closer analysis of the distal fall-off region, all the curves seem to intersect at a common range point close to $\mathrm{R}_{80}$, as shown in the insert at the upper left corner of Figure 7. In other words, the $80 \%$ dose range of a beam does not change with varying initial energy spread. This finding is consistent with that reported by others. ${ }^{[2,11]}$

\section{Conclusion}

PTSim MC simulations were conducted to study depth dose characteristics of ideal proton beams within therapeutic energy ranges. Under the assumption of charged particle equilibrium, study results successfully demonstrated the effectiveness of the PBG technique in depth dose simulation. Energy-range relationships were obtained for range parameters of $\mathrm{R}_{\text {peak }}, \mathrm{R}_{90}, \mathrm{R}_{80}$ and $\mathrm{R}_{50}$ and their fitting formulae to third-order polynomial were provided in Table 1. These formulae can serve as handy reference for future study. For the effect of initial energy spread on BP shape, it was shown that FWHM may be a more sensitive index than the distal fall-off $\left(\mathrm{W}_{80-20}\right)$ in optimizing initial beam energy spread when performing MC simulation. It was also demonstrated that initial energy spreads had minimum effect on $R_{80}$ such that $R_{80}$ can be used as a good index to represent the initial incident mean beam energy.

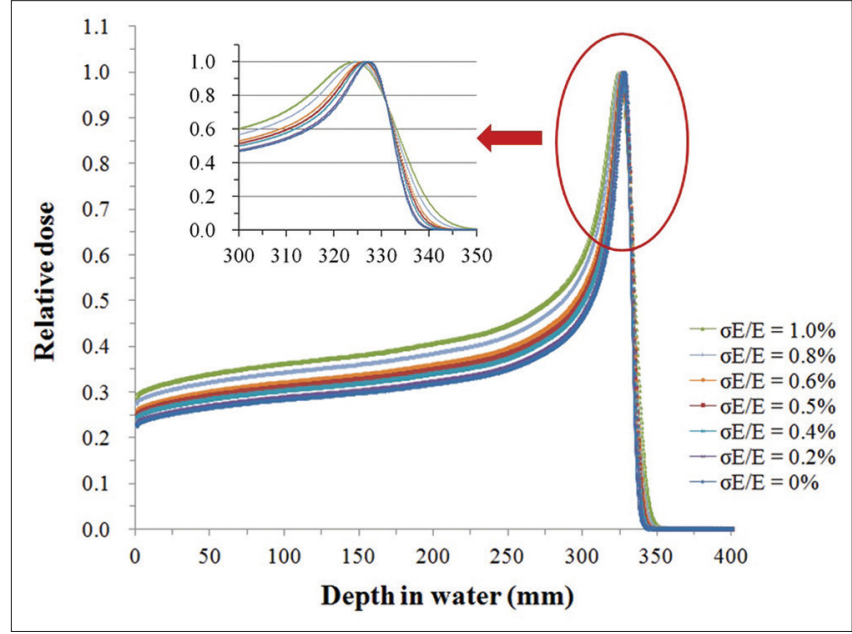

Figure 7: Similar depth dose plots as in Figure 6 for $230 \mathrm{MeV}$ beams with $0.1-1 \%$ initial energy spreads at $0.2 \%$ interval. Shown in the insert is the enlarged view near the distal end of the peaks. All curves intersect at a fixed range close to $R_{80}$.

\section{Acknowledgments}

This work was supported by the Chang Gung Research Project CMRPD180183, CMRPD1C0671 and CMRPD1C0672 for Proton Therapy Basic Research.

\section{Conflicts of interest}

There are no conflicts of interest.

\section{REFERENCES}

1. Berger M, Coursey J, Zucker M, Chang J. Stopping-Power and Range Tables for Electrons, Protons, and Helium Ions, NISTIR 4999, National Institute of Standards and Technology; May, 2015. Available from: http://www.nist.gov/pml/data/star. [Lst accessed on 2015 Sep 17].

2. Paganetti H. Proton Therapy Physics. Boca Raton, FL: CRC Press; 2011.

3. Aso T, Kimura A, Kameoka S, Murakami K, Sasaki T, Yamashita T. GEANT4 Based Simulation Framework for Particle Therapy System. In Nuclear Science Symposium Conference Record, 2007 NSS'07 IEEE: 2007. IEEE; 2007. p. 2564-7.

4. Agostinelli S, Allison J, Amako KA, Apostolakis J, Araujo H, Arce P, et al. GEANT4 - A simulation toolkit. Nucl Instrum Methods Phys Res A 2003;506:250-303.

5. Aso T, Yamashita T, Akagi T, Kameoka S, Nishio T, Murakami K, et al. Validation of PTSIM for Clinical Usage. In Nuclear Science Symposium Conference Record (NSS/MIC), 2010 IEEE: 2010. IEEE; 2010. p. 158-60.

6. Akagi T, Aso T, Iwai G, Kimura A, Kameoka S, Lee SB, et al Geant4-based particle therapy simulation framework for verification of dose distributions in proton therapy facilities. Prog Nucl Sci Technol 2014;4:896-900.

7. GEANT4 Collaboration, "Physics Reference Manual" [Online], Available from: http://geant4.cern.ch/support/userdocuments.shtml. 
[Last accessed on 2015 Sep 17].

8. Pedroni E, Scheib S, Böhringer T, Coray A, Grossmann M, Lin S, et al. Experimental characterization and physical modelling of the dose distribution of scanned proton pencil beams. Phys Med Biol 2005;50:541-61.

9. Lee C, Lee Y, Tung C, Cheng H, Chao T. MCNPX simulation of proton dose distribution in homogeneous and CT phantoms. Radiat Phys Chem 2014;95:302-4.

10. Paganetti H, Jiang H, Parodi K, Slopsema R, Engelsman M. Clinical implementation of full Monte Carlo dose calculation in proton beam therapy. Phys Med Biol 2008;53:4825-53.

11. Bortfeld T. An analytical approximation of the Bragg curve for therapeutic proton beams. Med Phys 1997;24:2024-33.

This is an open access article distributed under the terms of the Creative Commons Attribution-NonCommercial-ShareAlike 3.0 License, which allows others to remix, tweak, and build upon the work non-commercially, as long as the author is credited and the new creations are licensed under the identical terms.

For reprints contact: reprints@ medknow.com 